\title{
Immunomodulatory and anti-inflammatory properties of macrolides
}

\author{
Magdalena Bulska*, Daria OrszulaK-Michalak
}

Chair and Department of Biopharmacy, Faculty of Pharmacy, Medical Univeristy of Lodz, Muszynskiego 1, 90-151 Lodz, Poland

\begin{tabular}{l}
\hline ARTICLE INFO \\
\hline Received 13 February 2014 \\
Accepted 25 March 2014 \\
\hline
\end{tabular}

\section{Keywords:}

antibiotics,

immunomodulation,

inflammation,

macrolides.

\begin{abstract}
Macrolides are a group of antibiotics whose activity is ascribable to the presence of the macrolide ring, to which one or more deoxy sugars may be attached. Two properties are inherent in this group of antibiotics, the immunomodulatory and the anti-inflammatory actions, ensuring great efficacy in a wide spectrum of infections. Macrolides demonstrate several immunomodulatory activities both in vitro and in vivo. They can down-regulate prolonged inflammation, increase mucus clearance, prevent the formation of bacterial biofilm and either enhance or reduce activation of the immune system. According to given properties and exceptional effects on bacterial phatogens, the macrolide antimicrobial agents have been found to serve a unique role in the management of chronic airway disorders, including diffuse panbronchiolitis, cystic fibrosis and chronic obstructive pulmonary disease. Use of macrolides can result in clinical improvement in patients with severe, chronic inflammatory airway diseases, improving their spirometry indicators, gas exchange and overall quality of life.
\end{abstract}

\section{INTRODUCTION}

Macrolides are a group of antibiotics whose activity is ascribable to the presence of the macrolide ring, a large macrocyclic lactone ring, to which one or more deoxy sugars, usually cladinose and desosamine, may be attached. The most commonly used macrolides have 14 (e.g. erythromycin, clarithromycin, roxithromycin) or 15 (e.g. azithromycin) atoms attached to their macrocyclic ring and therefore defined as 14- or 15-membered ring macrolides [1]. Macrolides are used to treat infections caused by Grampositive bacteria or in case of Streptococcus pneumoniae and Haemophilus influenzae infections, such as respiratory tract and soft-tissue infections. They have also been shown to be effective against Legionella pneumophila, mycoplasma, mycobacteria, some rickettsias and chlamydia [11]. Macrolide antibiotics have good bioavailability by the oral route, superb tissue penetration, favorable side-effect profiles and prolonged tissue persistence. Macrolides act as antibacterials by reversibly binding to the $\mathrm{P}$ site on the $50 \mathrm{~S}$ subunit of bacterial ribosomes. This action is mainly bacteriostatic, but it can also become bactericidal at high concentrations.

\footnotetext{
Corresponding author

* e-mail: magdalena.bulska@umed.lodz.pl

tel.: +48426779121

mob.: 502-313-624
}

Macrolides can accumulate within leukocytes and are therefore transported into the site of infection. What is interesting, two properties are inherent in this group of antibiotics, namely the immunomodulatory and the anti-inflammatory actions, ensuring great efficacy in a wide spectrum of infections [12].

\section{Anti-inflammatory and immunomodulatory effects}

Macrolides have a direct antimicrobial effect but more importantly, also modulate many components of the immune response. Because of this anti-inflammatory or immune modulating effect, macrolide antibiotics have been widely used as a maintenance treatment for various chronic inflammatory airway diseases [1]. Interest in the immunomodulatory effects of macrolides began with showing that in patients with bronchial asthma, requiring glucocorticoids administration, application of macrolide antibiotics allowed for reducing steroids dose [6]. This phenomenon is known as 'sparing effect'. Small doses of macrolide, independently from glucocorticoids action, reduce bronchial hyperreactivity in patients with severe asthma. Furthermore, it was shown that macrolides treatment increases the survival time in patients with diffuse panbronchiolitis (DPB). After 1987, when erythromycin was introduced as a standard therapy for DPB, an impressive increase in 10-year survival was seen. Previous studies have indicated that the effectiveness of such treatment is limited to 14- and 15-membered macrolides [7]. 
Among the commonly used macrolides, azithromycin, clarithromycin and erythromycin, are thought to have the greatest immunomudulatory activity [9].

Macrolides demonstrate several immunomodulatory activities both in vitro and in vivo. They can down-regulate prolonged inflammation, increase mucus clearance, prevent the formation of bacterial biofilm, and enhance or reduce activation of the immune system. They may also influence phagocyte activity by modyfying their miscellaneous functions (such as chemotaxis, phagocytosis, oxidative burst, bacterial killing, cytokine production) [12]. Macrolides decrease the production of reactivity oxygen species, inhibit neutrophil activation and mobilisation, accelerate neutrophil apoptosis, and block the activation of nuclear transcription factors [12].

An integral role in the initiation, perpetuation and subsequent down-regulation of the immune response is played by cytokines. Proinflammatory cytokines (e.g. interleukin IL-1, IL-2, IL-4, IL-6, IFN- $\gamma$, TNF- $\alpha$ ) and chemokines a subgroup of cytokines that affect chemotaxis - (e.g. IL-8) amplify the immune response through positive feedback loops. Anti-inflammatory cytokines, prostaglandins and transforming growth factor attenuate the immune response through a negative feedback mechanism. In general, macrolides inhibit the synthesis and/or secretion of proinflammatory cytokines while increasing the release of anti-inflammatory cytokines. Changes in cytokine and chemokine production are probably related to an effect of macrolides on the activation of transcription factors and activator protein [3].

Additionally, macrolides promote the phagocytosis of apoptotic cells by alveolar macrophages, thus avoiding secondary necrosis and releasing the cell contents that may induce further inflamation. Macrolides are also said to promote monocyte-to-macrophage differentiation, thereby increasing the number of active macrophages [5].

The most distinct effect of macrolide antibiotics is found in neutrophils, the key players of the anti-inflammatory response. Firstly, macrolide antibiotics cause a significant reduction in the chemotactic response of neutrophils to chemokines, which results in markedly decreased airways neutrophilia in patients with various inflammatory pulmonary diseases. Secondly, macrolides seem to stimulate exocytosis, and as a result enhancing the antibactericidal activity. Another anti-inflammatory effect of macrolide antibiotics is reducing the release of adhesion molecules in bronchial epithelial cells. Furthermore, macrolides shorten neutrophil survival by accelerating neutrophil apoptosis [1].

Interesting properties of macrolides is their inhibitory effect on biofilm formation by certain species of pathogens, such as Pseudomonas aeruginosa, which is one of the most virulent biofilm-forming microorganisms with a natural resistance to macrolides. The effect of macrolides on biofilm formation was also demonstrated in Haemophilus influenzae and Staphylococcus epidermidis [1]. Inhibition of bacterial biofilm formation is an action from the borderline of bactericidal or bacteriostatic and immunomodulatory properties. The biofilm is formed on the surface, which is infected or colonized by the proteins and mucopolysaccharides, produced by bacteria. It hinders the access of given antibiotics, thereby contributing to frequent recurrence of infections. One of the proteins responsible for the biofilm formation is flagellin. It has been shown that macrolides, such as erythromycin, clarithromycin, azithromycin, in concentration much lower than minimum inhibitory concentration required to inhibit the growth of $50 \%$ of microorganisms $\left(\mathrm{MIC}_{50}\right)$, inhibit the production of flagellin. As a consequence, macrolides impede biofilm preparation and reduces the motility of bacteria [10].

Over the past few years, numerous studies have documented that certain macrolides have a wide spectrum of immunomodulatory effects on mammalian cells. These effects limit tissue damage by neutrophils, decrease mucus viscosity and suppress angiogenesis. For clarity, specific effects can be categorized on: host-pathogen interactions, signaling pathways, cytokine responses, oxidative stress, innate immunity and other (Tab. 1). Additionally, immunomodulatory effects of macrolides support the rationale for using them in therapy for the treatment of chronic inflammatory airway diseases [4].

Table 1. Immunomodulatory effects of macrolides [4]

\begin{tabular}{|c|c|}
\hline Category & Specific Effects \\
\hline $\begin{array}{l}\text { Host-pathogen } \\
\text { interactions }\end{array}$ & 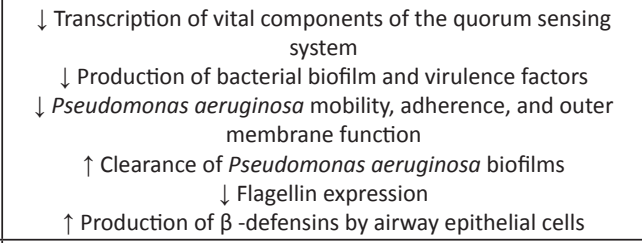 \\
\hline $\begin{array}{l}\text { Signaling } \\
\text { pathways }\end{array}$ & $\begin{array}{c}\text { Inhibits NF- } \mathrm{KB} \text { and AP-1 activation pathways in bronchial } \\
\text { epithelial cells } \\
\downarrow \text { MMP-9 expression and activity } \\
\downarrow \text { TNF- } \alpha \text { levels and secretion in CF-derived airway epithelial cells }\end{array}$ \\
\hline $\begin{array}{l}\text { Cytokine } \\
\text { responses }\end{array}$ & 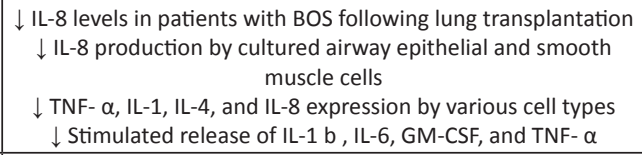 \\
\hline Oxidative stress & $\begin{array}{l}\downarrow \text { Superoxide and elastase release from stimulated human PMNs } \\
\text { Impairs the phagocytic oxidative burst of PMNs }\end{array}$ \\
\hline Innate immunity & $\begin{array}{c}\uparrow \text { Neutrophil, lymphocyte, histiocyte, and eosinophil apoptosis } \\
\downarrow \text { Neutrophil chemotaxis and survival }\end{array}$ \\
\hline Other & $\begin{array}{l}\quad \downarrow \text { Mucus secretion } \\
\downarrow \text { Methylprednisolone elimination }\end{array}$ \\
\hline
\end{tabular}

$\mathrm{AP}$ - activator protein; $\mathrm{BOS}$ - bronchiolitis obliterans syndrome; $\mathrm{CF}$ cystic fibrosis; GM-CSF - granulocyte-macrophage colony- stimulating factor; $\mathrm{MMP}$ - matrix metalloproteinase; NF- $\mathrm{KB}$ - nuclear factor $\kappa$ - lightchain-enhancer of activated B cells; PMNs - poly- morphonuclear leukocytes; TNF- $\alpha$ - tumor necrosis factor $\alpha$

\section{Usefulness of immunomudulatory actions in clinical practice}

According to given immunomodulatory properties and unique effects on bacterial pathogens, macrolide antimicrobial agents have been found to have the potential to serve a unique role in the management of chronic airway disorders. Subsequently, macrolides have been studied | for different airway diseases including DPB, CF and chronic obstructive pulmonary disease (COPD). The available data confirm that using macrolides can result in clinical improvement in patients with severe, chronic inflammatory airway diseases associated with frequent bacterial colonization and chronic infection. 
a) Effectiveness of immunomodulatory properties of macrolides in diffuse panbronchiolitis

The most convincing demonstration of the immunomodulatory effects of macrolides has been shown in the DPB treatment, a pulmonary disease of unknown etiology that was found primarily in Japan. The application of the macrolides has resulted in a dramatical increase of the 10-year survival rate of patients, i.e. from $26 \%$ to over $90 \%$ [7]. The effectiveness of these drugs appears to be limited to the 14and 15-membered macrolides. Beneficial results have been confirmed with erythromycin, clarithromycin, roxithromycin and azithromycin. They improve pulmonary function and decrease morbidity and mortality in patients with DPB. Moreover, they reduce proinflammatory cytokines in serum and bronchoalveolar lavage (BAL) fluid, decrease mucus hypersecretion and may protect the airway epithelium from damage. Those effects are unlikely to be a pure result of the antibacterial activity of the macrolide, because the maximal serum and sputum levels of erythromycin have been documented to be below the MICs of the clinically identified isolates. Macrolides, used in smaller doses than in case of infections, improved spirometry indicators and gas exchange in patients lungs in the first six month of therapy, however they are well tolerated even after several years of treatment $[8,10]$.

\section{b) Benefits of using macrolides in cystic fibrosis}

CF is largely similar to DPB, including symptoms and pulmonary pathology. Both deseases are characterized by cough, persistent sinus disease, neutrophilic airway inflammation, susceptibility to persistent endobronchial infection with opportunistic pathogens, and progressive deterioration in pulmonary function. Besides, both diseases are responsive to the immunomadulatory effects of macrolides [1].

$\mathrm{CF}$ is an autosomal recessive disorder, characterized by chronic airway infection, mainly with Staphylococcus aureus, Haemophilus spp. and Pseudomonas aeruginosa. It is caused by a defect in the CF transmembrane regulator. Alterations in epithelial cell ion transport, as a result of defective CF transmembrane regulator, lead to increased sputum viscosity causing stasis of secretions, recurrent respiratory infections, and chronic progressive bronchiectasis. Long-term effects of the bronchiectasis include impaired mucociliary clearance, chronic, acute infection and inflammation, structural airway and parenchymal injury accounting for much of the morbidity and mortality associated with the disease. The airway inflammatory response in patients with $\mathrm{CF}$ is predominately neutrophilic. Moreover, an increase of proinflammatory cytokine production is observed [10].

The possibility that macrolides may modify the neutrophilic inflammatory response in CF was first investigated in the early 2000s. Now, a number of studies have demonstrated beneficial effects in both adults and children. Patients receiving macrolides, especially azithromycin, have improved their spirometry and quality of life $[4,12]$.

There are several theoretical premises why macrolide antibiotics could be disease-modifying agents in CF. First, airway inflammation is recognized as a major factor in the pathogenesis of lung disease in CF. Macrolides at high doses have been shown to retard the decline of lung function in CF. Secondly, macrolides reduce sputum viscoelasticity and airway adhesion of Pseudomonas aeruginosa. Furthermore, they decrease inflammatory response in CF. In addition to the anti-inflammatory and anti-bacterial effects, macrolides may also directly alter Pseudomonas aeruginosa biology, f.e. by reducing the organism's ability to produce virulence factors [4].

To summarize, studies in patients with CF indicate that long-term use of low-dose macrolides, azithromycin mainly, improves clinically relevant end points, nevertheless the optimum dosage and time administration are still under investigation $[4,10,12]$.

\section{c) Potential advantages of using macrolides in chronic} obstructive pulmonary disease patients

The application of the macrolide antibiotics have assumed an important role in the management of COPD. Mainly because of their broad spectrum coverage and excellent safety profile, but also their potent anti-inflammatory effects. The Global Burden of Disease study has concluded that COPD will become the third leading cause of death worldwide by 2020 [8].

Acute exacerbations of COPD are associated with impaired quality of life and pulmonary function. Airway and lung parenchyma inflammation are now known to play an important role in COPD. Both neutrophil and eosinophil activation and recruitment have been observed, while several inflammatory mediators are involved in the inflammatory cascade. A variety of microbes stimulates the inflammatory process and precipitate acute exacerbations of COPD, which may increase the rate of lung function decline that occurs over time. Given the importance of inflammation and bacterial infection in the pathogenesis of COPD, it is evident that macrolides may offer unique advantages as diseasemodifying agents and such effect may be multifactorial. However, some subsequent studies need to be carried out in order to answer the question, whether it is caused by their antimicrobial activity, immunomodulation or both $[4,8,12]$. Potential beneficial effects of macrolides in COPD patients are presented below (Fig. 1) [8].

The role of macrolide therapy in COPD patients requires additional study to explain better several issues, such as:

- Do macrolides attenuate baseline inflammation or do they prevent or attenuate acute exacerbations of COPD episodes?

- What is the effect of treatment of chronic macrolide therapy in COPD patients treated with conventional therapies?

- Does the therapeutic effect reflect an antimicrobial activity, an immunomodulatory effect, or both?

- What is the toxicity of chronic therapy in COPD patients?

- What is the risk - benefit of macrolide therapy in COPD patients?

Considering these vital aspects requires additional, welldesigned, placebo-control trials. Finding answers to above questions is a necessary condition before using the macrolide prophylaxis in COPD patients on a larger scale [8]. 


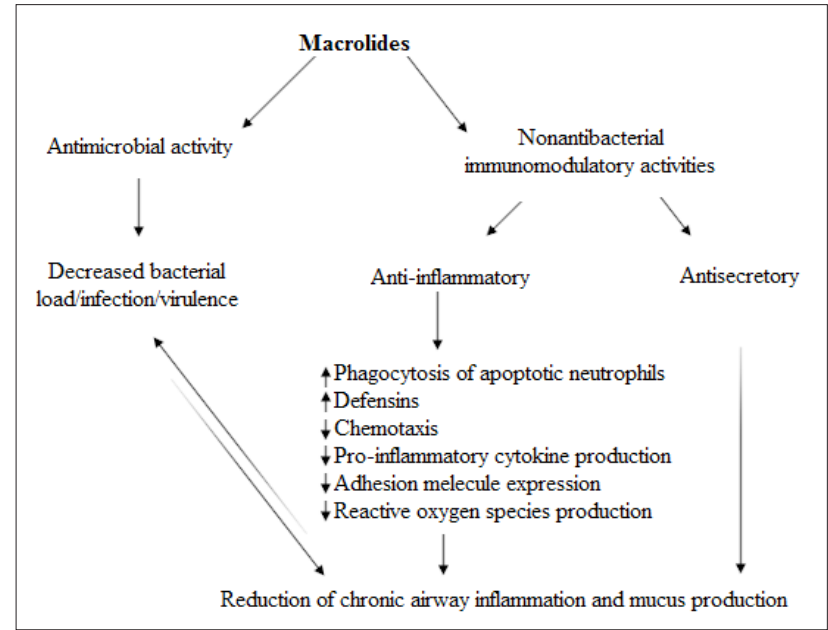

Figure 1. Potential beneficial effect of macrolides in COPD patients [8].

\section{CONCLUSION}

After more than 30 years, macrolides still hold a vital place in our therapeutic armamentarium. They possess immunomodulatory and anti-inflammatory actions extending their antibacterial activity. Indeed, they are able to suppress the "cytokine storm" of inflammation and confer an additional clinical benefit through their immunomodulatory properties. The majority of cells, involved in both the innate and adaptive immune responses, are influenced when macrolide antibiotics are administered. The most distinct effect of macrolides is found in neutrophils. Neutrophil accumulation, adhesion and apoptosis are clearly reduced, which results in markedly decreased airway neutrophilia. In respiratory diseases, macrolides have so far manifested variable efficacy, but we still need to define dose and duration of administration. Moreover, we also need to find out which macrolide antibiotic may be proved superior in each condition. As for potential future research, it seems that there is some ground for analyzing the role of macrolides in the treatment of respiratory diseases, which may be enhanced by creating agents with a profound anti-inflammatory effect and little antibacterial effect. According to a concept that has been investigated in tetracyclines, immunomodulatory macrolides devoid of anti-infective properties are developed by modifying the molecular structure of the atoms attached to the macrocyclic ring. These purely immunomodulatory macrolides would offer a way to circumvent bacterial resistance and lead to the development of more specific therapeutic agents $[2,12]$.

\section{REFERENCES}

1. Altenburg J. et al.: Immunomodulatory effects of macrolide antibiotic-part 1: biological mechanisms. Respiration, 81, 67, 2011.

2. Cazalias J., Tanabe S., Gagnon G.: Tetracyclines and chemically modified tetracycline-3 (CMT-3) modulate cytokine secretion by lipopolysaccharide-stimulated whole blood. Inflammation, 32, 130, 2009.

3. Culic O., Erakovic V., Parnham M.J.: Anti-inflammatory effects of macrolide antibiotics. Eur. J. Pharmacol., 429, 209, 2001.

4. Friedlander A.L., Albert R.K.: Chronic macrolide therapy in inflammatory airway diseases. Chest, 138, 1202, 2010.

5. Hodge S., Hodge G., Jersmann H.: Azithromycin improves macrophage phagocytic function and expression of mannose receptor in chronic obstructive pulmonary disease. Am. J. Respir. Crit. Care Med., 178, 139, 2008.

6. Itkin I.H., Menzel M.L.: The use of macrolide antibiotic substances in the treatment of astma. J. Allergy, 45, 146, 1970.

7. Kudoh S., Azuma A., Yamamoto M.: Improvement of survival in patients with diffuse panbrochiolitis treated with low-dose erythromycin. Am. J. Respir. Crit. Care Med., 157, 1829, 1998.

8. Martinez F., Curtis J., Albert R.: Role of macrolide therapy in chronic obstructive pulmonary disease. Interantional Journal of COPD, 3 , 331, 2008.

9. Rubin B.K., Henke M.O.: Immunomodulatory activity and effectiveness of macrolides in chronic airway disease. Chest, 125, 70, 2004.

10. Targowski T., Jahnz-Różyk K.: Immunomodulatory properties of macrolides. Pol. Merk. Lek., 149, 408, 2008.

11. Ungureanu V.: Macrolides, lincosamides, streptogramines (MLS): mechanisms of action and resistance. Bacteriol Virusol Parazitol Epidemiol., 55, 131, 2010.

12. Zarogoulidis P. et al.: Macrolides: from in vitro anti-inflammatory and immunomodulatory properties to clinical practise in respiratory diseases. Eur. J. Clin. Pharmacol., 68, 479, 2012. 